\title{
The early activity of Ileana on Nuclear Physics: reactions induced by nucleons
}

\author{
A. Bracco $^{1}$ \\ Dipartimento di Fisica, Università degli Studi di Milano and INFN Sezione di Milano \\ Via Celoria 16, 20133 Milano, Italy \\ E-mail: angela.bracco@mi.infn.it \\ M. Pignanelli \\ Dipartimento di Fisica, Università degli Studi di Milano \\ Via Celoria 16, 20133 Milano, Italy \\ E-mail: marcello.pignanelli@mi.infn.it
}

\begin{abstract}
The research activity of Ileana, from 1956 to 1980, is briefly reported. The focus is on a number of relevant results that she obtained with her dedicated work and that were at the frontier of research in Nuclear Physics. They concern pioneer contributions to the building blocks of nuclear reaction mechanisms: evaporative processes, statistical fluctuations, dynamics of the two- and three-nucleon transfers reactions and of pre-equilibrium processes.
\end{abstract}

XLVIII International Winter Meeting on Nuclear Physics - BORMIO2010

Bormio, Italy

January 25-29 2010

\footnotetext{
$1 \quad$ Speaker
} 


\section{Introduction}

Ileana graduated in Physics in 1954 at the University of Modena, where she was involved in experiments on cosmic rays observed by nuclear emulsions [1]. She then began her training in Nuclear Physics in 1956/57 in Milan, participating in systematic studies providing the basis of the thermal behavior of the nuclear system. The work made on compound nucleus reactions showed evaporative processes described with the statistical model and gave information on nuclear level densities as deduced from evaporative spectra [2-7] and on level widths from cross section fluctuations [8-13]. These studies were performed with small electrostatic accelerators, available in Milan and delivering, low energy deuteron beams used directly or to obtain $14 \mathrm{MeV}$ neutrons. In the following years (1965-1980), with the availability of proton beams from the Milan AVF Cyclotron [14], Ileana took part to systematic studies of nuclear reactions induced by protons and producing direct processes, with particular attention to the two- and threenucleon transfer reactions and on pre-equilibrium processes in nuclear dynamics [15-22].

During the years 80 's and 90' Ileana had collaborations with the experimental groups of Legnaro on heavy ions reactions with energy around the Coulomb barrier (dissipative processes, fusion-fission with low energy heavy ions collisions). After 1990 her main interest shifted gradually to the physics of heavy ion collisions at higher energies and she carried out experiments at different Laboratories, among which LBL, GANIL, MSU. Particularly important was her collaboration to the activities at LNS (Catania) and GSI (Darmstadt). The most important issues addressed with those researches were the liquid-gas phase transition and the modification of the properties of mesons in nuclear matter.

The following sections are intended to describe the early activity of Ileana, namely her research carried out in Milan from 1956 to 1980.

\section{The years from 1956 to 1965: neutron induced reactions and the statistical model}

Ileana obtained a position at CISE (Centro Informazioni Studi ed Esperienze), an institution that was originally born to perform research related to the nuclear energy production and specifically to the design of nuclear fission reactors. Due to major uncertainties in the national energy plans, no reactor was ever built or installed by CISE. However CISE gave the opportunity to perform a free, university style, scientific research. Indeed, excellent results were obtained in Nuclear Physics, Electronics, Material Science, Laser, etc. In CISE, in the years form 1956 to 1965, Ileana had a good training in experimental Nuclear Physics. Particularly significant for her was the close collaboration with Ugo Facchini, who promoted strongly Nuclear Physics first at CISE and then at the University of Milano. In those years she got a major imprinting on two specific topics: the mechanism of nuclear reactions and the associated nuclear instruments. The first publication of Ileana with Facchini [2] concerns the determination of the age of fossils and of mineral deposits by $\alpha$-emission detected in ionization chambers (Fig. 1). From the following years and up to recent time Ileana kept very well alive the interest in developing and using ionization chambers. In 1958 Ileana published her first paper [3] on nuclear reactions, within a collaboration Facchini, Colli, Marcazzan, Sona and Pignanelli. 

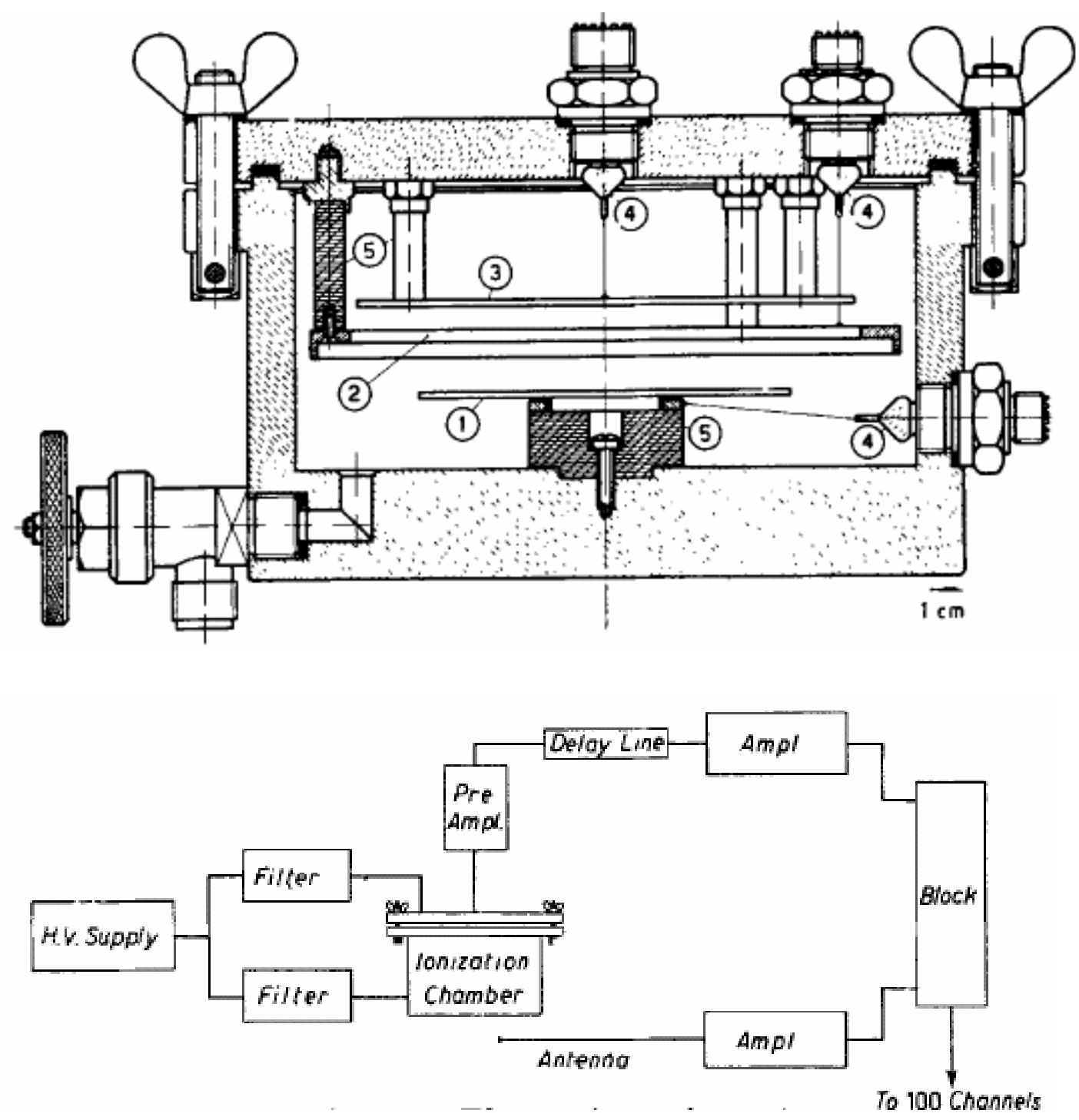

Figure 1- From Ref. [2]. The upper part shows a sketch of the Ionization Chamber used to measure alpha radioactivity at low concentration: 1) emitting electrode; 2) grid; 3) collector; 4) kovar-glass seals; 5) insulator. Below the electronic equipment is given. It should be noted in particular a 100 channels analyzer (very rare instrument at that time) and the inclusion of an antenna, used to veto the noise in the electronic signals produced by the public transportation passing near the laboratory. The passage of trams was detected by such antenna.

The paper reported the energy spectra of protons emitted in the $(n, p)$ reaction induced by $14 \mathrm{MeV}$ neutrons on several nuclei. The neutrons were produced by the $\mathrm{d}+\mathrm{t}=\mathrm{n}+\alpha$ reaction. The necessary deuteron beam (at a laboratory energy of $200 \mathrm{keV}$ ) was obtained with small Cockcroft-Walton accelerators (one at CISE and a second at the University). The flux of neutrons per second was of the order of 107 per second in total and less that 105 per second on 


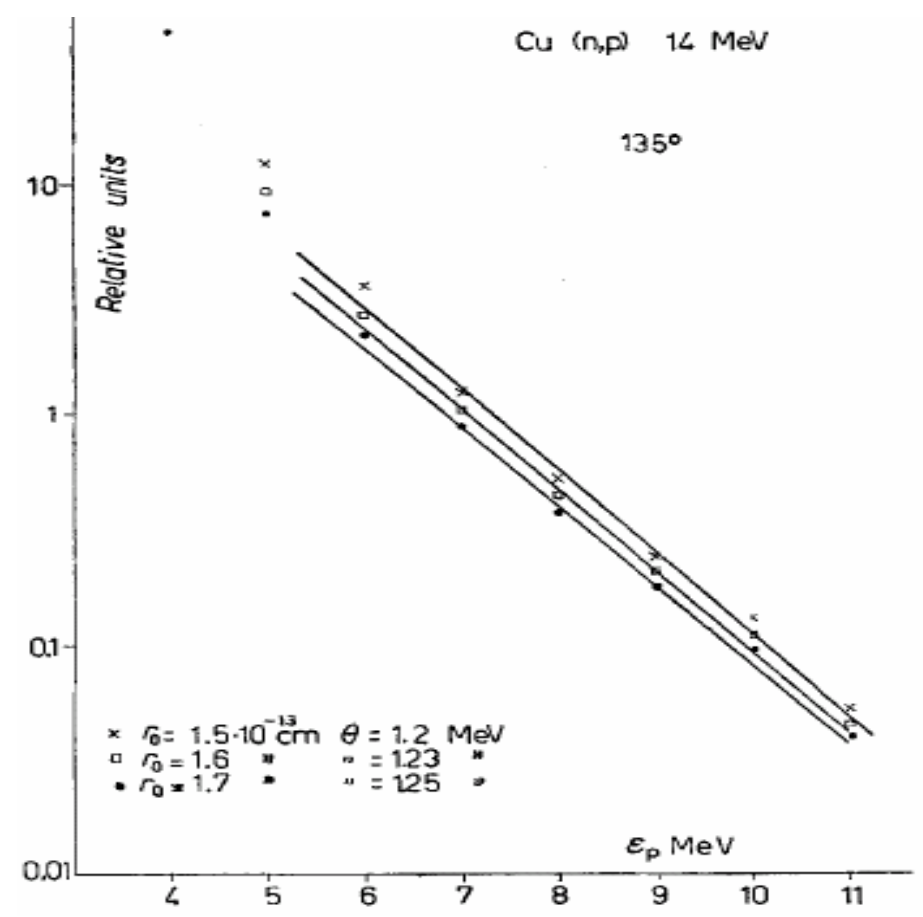

Figure 2 - From Ref. [6]. The nuclear temperature $\theta$ calculated from the backward angle spectra of the $(n, p)$ reaction on $\mathrm{Cu}$ at $14 \mathrm{MeV}$. The three curves are roughly represented with the expression $\exp [-\varepsilon / \theta]$. The indicated $\theta$ values were obtained using different Coulomb penetrabilities.

target. However the main difficulty was not due to the intensity, but to the presence of considerable background. The protons emitted in the reaction were detected by a $\mathrm{CsI}(\mathrm{Tl})$ scintillator, not shielded from neutrons. The stronger component of the background was due to protons produced directly by reactions induced by neutrons in the scintillator detector material. However, clean proton spectra, with a very low background, were obtained by improving progressively the detector system, which made use of coincidences between scintillating and proportional gas counters [4]. The background was also reduced by an accurate choice of the materials present in the detector system, taking advantage of the fact that the $(n, p)$ reaction in ${ }^{12} \mathrm{C}$ and ${ }^{16} \mathrm{O}$ is hindered by large negative $\mathrm{Q}$ values. For this reason the counter walls were made out of graphite and the gas employed in the proportional counters was $\mathrm{CO}_{2}$.

The proton spectra observed in these experiments display a continuous shape with an exponential slope. Comparisons with model predictions were made, dividing the proton counts $\mathrm{n}(\varepsilon)$ by the expression $\varepsilon \sigma_{\mathrm{C}}, \varepsilon$ being the energy of the emitted proton and $\sigma_{\mathrm{C}}$ the so called inverse cross-section. According to the statistical model the ratio $\mathrm{n}(\varepsilon) / \varepsilon \sigma_{\mathrm{C}}$, is proportional to the level density. This ratio, in the range from 4 to $10 \mathrm{MeV}$, is described fairly well, as shown in Fig. 2, by an exponential law $\mathrm{e}^{-\varepsilon / \theta}$, being $\theta$ the nuclear temperature. A better agreement, in a wider energy range, was obtained using, the expression for the density of nuclear levels originally introduced by Bethe and then developed by other authors as Lang and Le Couteaur [5]. In this expression the energy dependence of the level density is given by the factor $\exp [2 \sqrt{ }(a \mathrm{U})] /(\mathrm{U}+\mathrm{t})^{2}$ 
where $U$ is the internal energy of the nucleus (the excitation energy corrected for the pairing energy) and $t$ is the thermodynamic temperature. This latter temperature is obtained from the relation $\mathrm{U}=a \mathrm{t}^{2}-\mathrm{t}$, where the parameter $a$ gives the density of single particle states at the Fermi energy. The obtained results were summarized by Facchini-Iori-Menichella [6] in a paper in which it was shown that the evaporative model gives a satisfactory interpretation of (n,p), (n, n'), $(\mathrm{n}, \mathrm{np})$ and $(\mathrm{n}, 2 \mathrm{n})$ cross sections and finally by Erba-Facchini-Menichella [7] in a paper in which the energy spectra of particles emitted in $(n, p),(p, n)$ and $\left(n, n^{\prime}\right)$ reactions are analyzed by statistical model calculations. A rather smooth mass dependence of the parameter $a$, with oscillations at the shell closures, was evidenced. The $a$ values obtained from evaporative spectra were in good agreement with those from slow neutron resonance data.
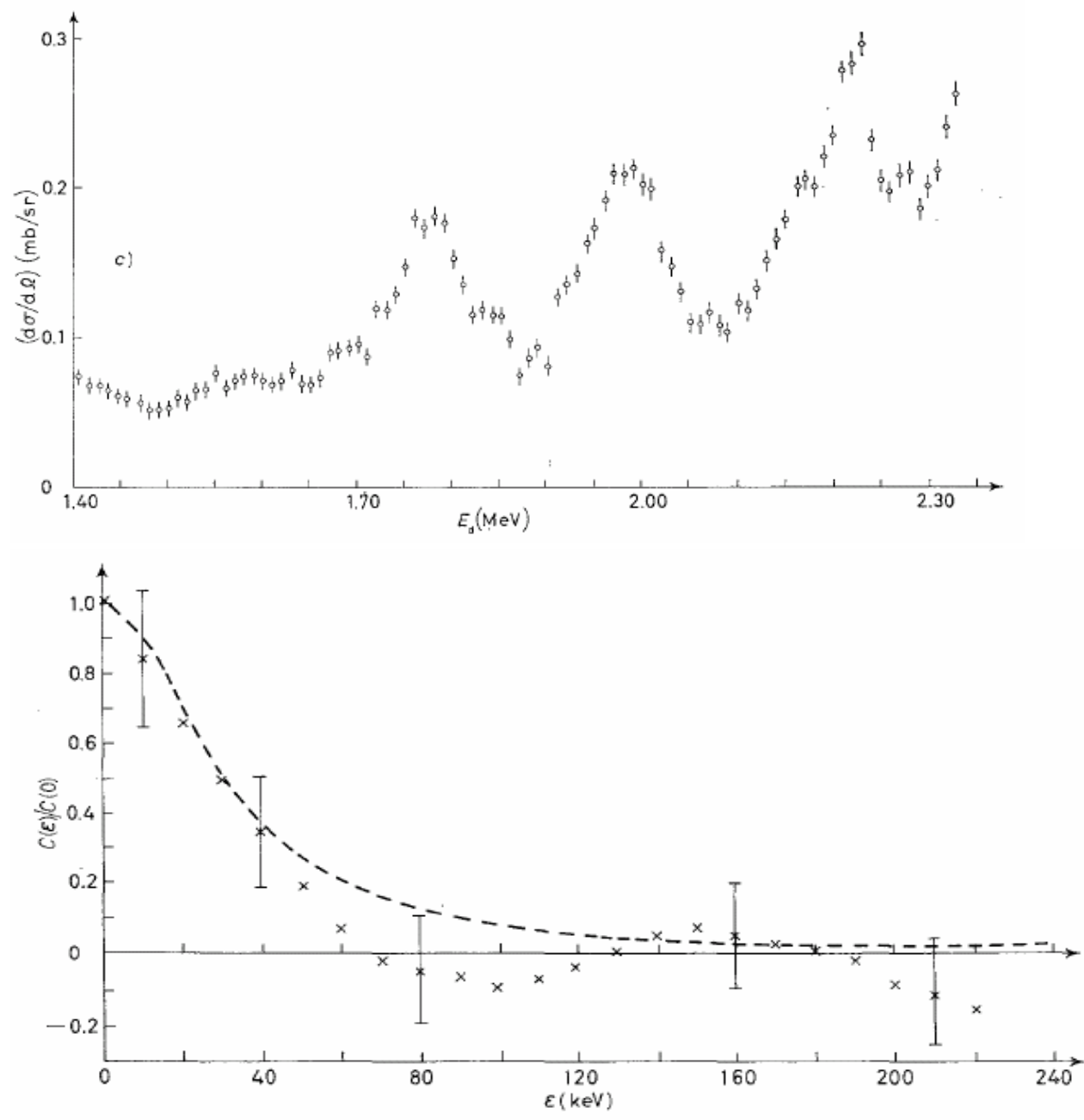

Figure 3. The statistical fluctuations (Ericson fluctuations) identified in the experiment described in Ref. [12]. Upper part: excitation function in the transition ${ }^{27} \mathrm{Al}\left(\mathrm{d}, \alpha_{2}\right)^{25} \mathrm{Mg}^{*}$. Lower part: autocorreletion function from the analysis of the excitation function of the $\alpha_{0}$ transition. The dashed curve is the calculated Lorentzian function with $\Gamma=30 \mathrm{keV}$. 
Before 1962 the phenomenology on statistical processes was mainly obtained from slow neutron resonances and from evaporative spectra. If $\Delta \mathrm{E}$ is the energy resolution, $\Gamma$ the level width and $\mathrm{D}$ the average distance between levels, slow neutron resonance measurements are characterized by the relation $\Delta \mathrm{E} \ll \Gamma \ll \mathrm{D}$. In contrast, the evaporative spectra measurements are characterized by $\Delta \mathrm{E} » \Gamma \gg \mathrm{D}$. In the latter case a smooth energy dependence of cross sections was predicted and observed. However, fluctuations around an average cross-section value were expected in the case of a well defined incident energy with respect to the level width $(\Delta \mathrm{E}<\Gamma)$, even in the continuum region $(\Gamma \gg \mathrm{D})$.

The first theoretical investigations on cross section fluctuations were carried out by Tolaf Ericson [8]. The first experimental evidence was obtained in 1962 in CISE from the reaction ${ }^{28} \mathrm{Si}(\mathrm{n}, \alpha)$ [9]. The ${ }^{28} \mathrm{Si}$ target was simply a Si-surface barrier detector, irradiated by $12.5-18.5$ $\mathrm{MeV}$ neutrons. These neutrons were obtained with the $\mathrm{d}+\mathrm{t}$ reaction, being the deuterons accelerated by a 3.5 MV van der Graaff. This electrostatic accelerator was constructed by Ileana with the help of only two engineers [10]. After this experiment Ileana participated in other studies on cross section fluctuations. The experimental data were collected at Saclay and in Milan. Significant studies were published in the papers [11-13], reporting also the methods to be used in the analysis of data in order to obtain correct values of the level widths (Fig. 3). In those years a long standing collaboration of Ileana with Ettore Gadioli was established.

The studies of statistical fluctuations promoted the organization of the Winter Meeting on Nuclear Physics. In fact, the first Meeting was held at Vilar (Switzerland) in 1963 under the scientific direction of Facchini and Ericsson. It was mainly devoted to nuclear reaction mechanisms and in particular to statistical processes. In 1973 the Meeting was moved in Bormio under the direction of Ileana, still with nuclear dynamics and reaction mechanisms as main topics.

\section{1965-1980: reactions induced by proton beams, direct and statistical effects}

In 1965 Ileana left CISE and moved to the Physics Institute of the University of Milano, and there she worked in strong collaboration with Pignanelli, Micheletti and Gadioli. In the same year the initial operation of the Milan AVF cyclotron started. This was the first AVF cyclotron in Europe and the second in the world, just after the Berkeley AVF cyclotron. It was designed by Succi, Resmini, Acerbi and et al. [14]. Due to the lack of funds, to save money, they simplified drastically the project. For instance, the radio frequency generator, already used for a transmitting radio station, was working at a fixed frequency. As a consequence the cyclotron could accelerate only protons, to a maximum energy of $45 \mathrm{MeV}$. Very soon it was found an original acceleration method and an efficient and fast beam extraction. An internal negative beam of $\mathrm{H}^{-}$ions was accelerated and then stripped off by a thin $\mathrm{Al}$ foil. After the stripping foil, the resulting $\mathrm{H}^{+}$ions, bent by the magnetic field and switched off from the cyclotron, gave the external proton beam. The energy of the extracted beam was fixed by the position of the stripping foil and was in the range of 14-45 MeV. After momentum analysis the energy spread could be reduced down to $20 \mathrm{keV}$. The above energy interval was interesting to 
study the interplay between the different reaction mechanisms: direct, pre-equilibrium and compound nucleus processes.

For more than 15 years the Milan AVF cyclotron, besides some applied physics and nuclear spectroscopy study, was intensively used for the following nuclear physics topics:

i) $\quad(p, \alpha)$ reactions: direct transitions, pre-equilibrium processes, compound nucleus.

ii) $\quad(p, t)$ and $\left(p,{ }^{3} \mathrm{He}\right)$ reactions: one- and two-steps processes in 2-nucleon transfers.

iii) Inelastic scattering and collective couplings.

iv) $\quad(p, x n)$ reactions: compound nucleus and pre-equilibrium components.

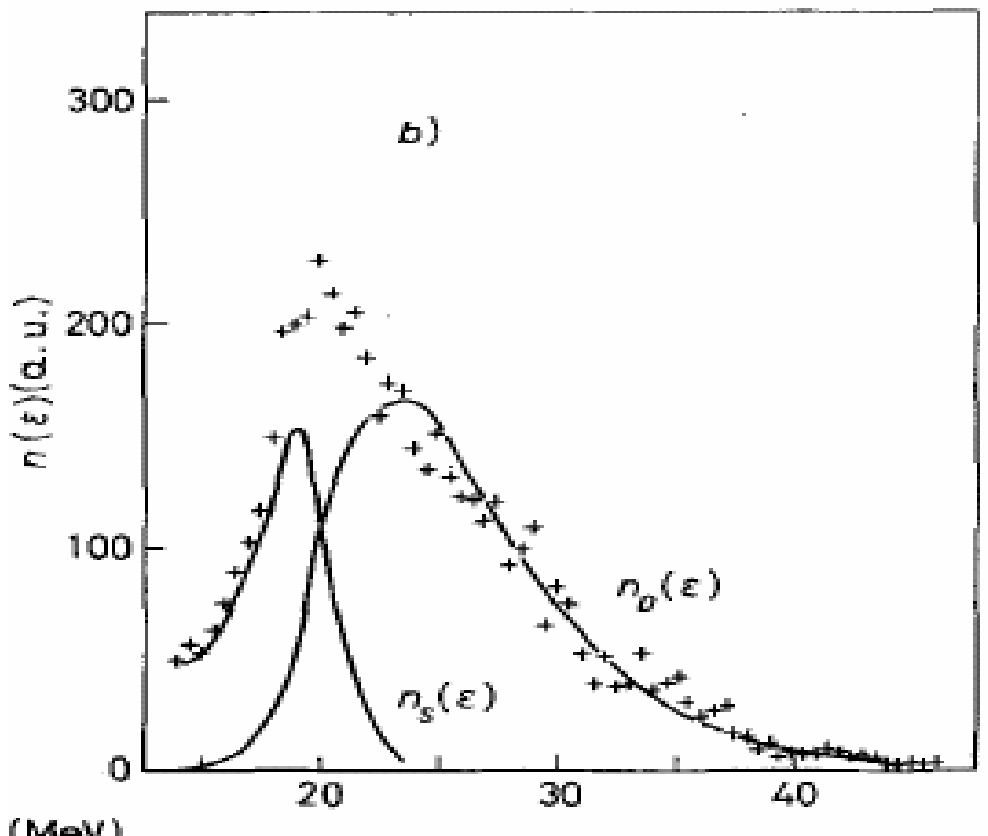

Figure 4 - Spectrum from the ${ }^{208} \mathrm{~Pb}(\mathrm{p}, \alpha)$ reaction, $\mathrm{E}=44 \mathrm{MeV}, \theta=150^{\circ}$ Decomposition of backward-angle spectrum in a pre-equilibrium, $\mathrm{n}_{\mathrm{D}}(\varepsilon$,$) and a statistical contribution, \mathrm{n}_{\mathrm{S}}(\varepsilon)[16]$.

Ileana participated actively in the first two types of experiments. The continuous spectra of the $\alpha$ particles emitted by reactions induced by protons on several medium mass and heavy nuclei were measured and analysed with the models describing the compound nucleus and preequilibrium processes. From the analysis of backward angle spectra the statistical contribution to the reaction was evaluated. Agreement between theoretical and experimental absolute values was found [15]. The shell effects on the level density in doubly magic nuclei, were evidenced in the analysis of $(\mathrm{p}, \alpha)$ data for $\mathrm{Bi}$ and $\mathrm{Pb}$ isotopes [16] and pre-equilibrium effects were observed and evaluated in the framework of the exciton model [16,17], as shown in Fig. 4.

The $(p, \alpha)$ transitions to low-lying states in the residual nucleus were also studied for many light nuclei. The differential cross sections display angular distributions mostly with a well developed diffraction pattern, typical of a surface, one-step interaction (Fig. 5). Attempts were made to describe the experimental angular distributions taking into account different direct mechanisms (t pick-up or $\alpha$ knock-out and possibly heavy-particle pick-up and knock-out) 
$[18,19]$. The main difficulty was found in the understanding of the energy dependence of the measured cross sections, that was not reproduced by DWBA calculations, while it was better described in the framework of the pre-equilibrium decay model, since the dependence of the experimental cross sections on the excitation energy was that typical of a compound system formation (right part of Fig. 5). Nevertherless, this pre-equilibrium model could not reproduce the angular distributions [20]. This was a puzzling result, pointing out the need of better direct reaction calculations. The difficulties found in these calculations were probably due to the ambiguities in the above direct mechanisms and to other uncertainties as those in transition form factors and in the poor matching conditions.
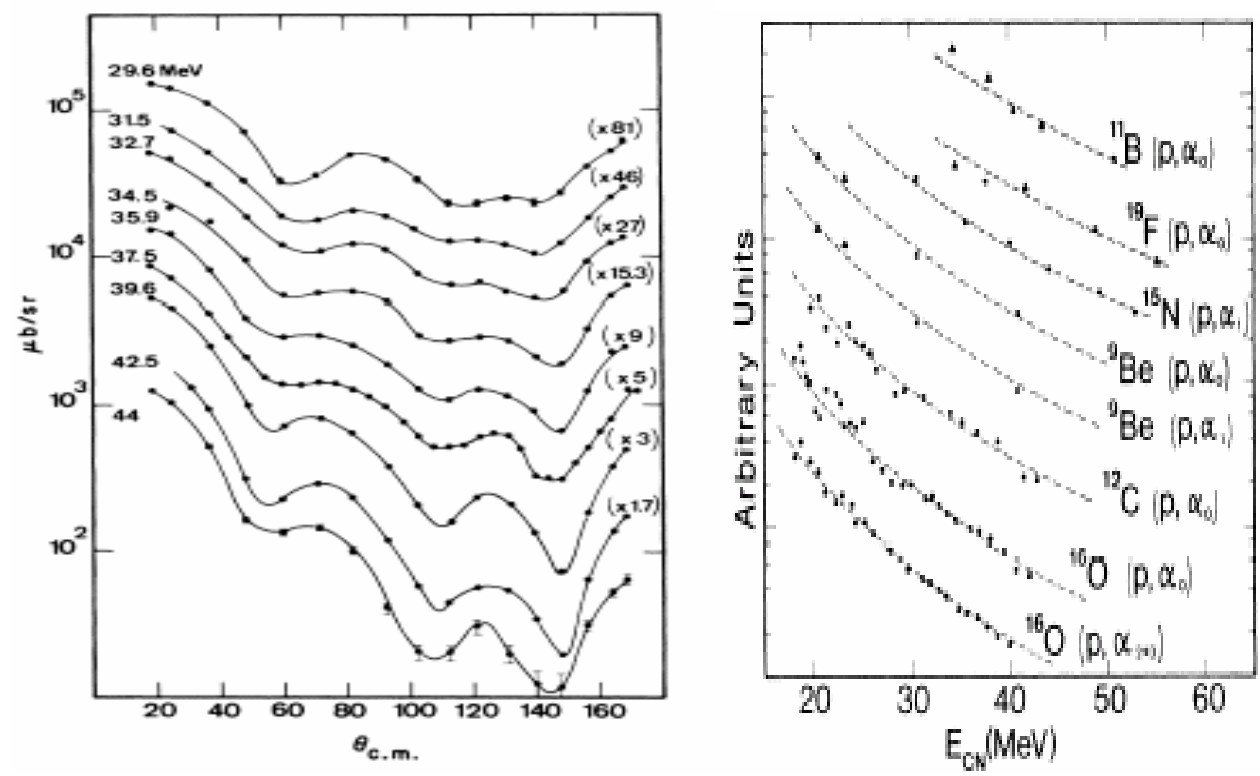

Figure 5 - Data from Ref. [20]. On the left: experimental differential cross sections for the reaction ${ }^{12} \mathrm{C}(\mathrm{p}, \alpha){ }^{9} \mathrm{~B}_{\text {g.s. }}$. On the right: mean energy dependence of $(p, \alpha)$ cross sections as a function of compound-system excitation energy. All dashed curves correspond to $\mathrm{E}^{-3.75}$

The differential cross sections for $(\mathrm{p}, \mathrm{t})$ and $\left(\mathrm{p},{ }^{3} \mathrm{He}\right)$ reactions on light nuclei [21] and in some medium mass nucleus [22] were measured at several incident proton energies. The data were compared with DWBA predictions. The energy dependence of the cross sections was poorly explained by calculations based on single-step 2-nucleon pick-up which gave a maximum in the cross section in the energy region where the angular momentum matching conditions are achieved. The absence of corresponding maxima in the experimental excitation functions were ascribed to other reaction mechanisms contributing substantially to the cross sections at low energies. The effect of the two-step processes was investigated. It was shown (Fig. 6) that successive single-neutron pick-up, $(\mathrm{p}, \mathrm{d}, \mathrm{t})$, can provide a satisfactory description of the energy dependence of the $(p, t)$ cross sections [22]. 


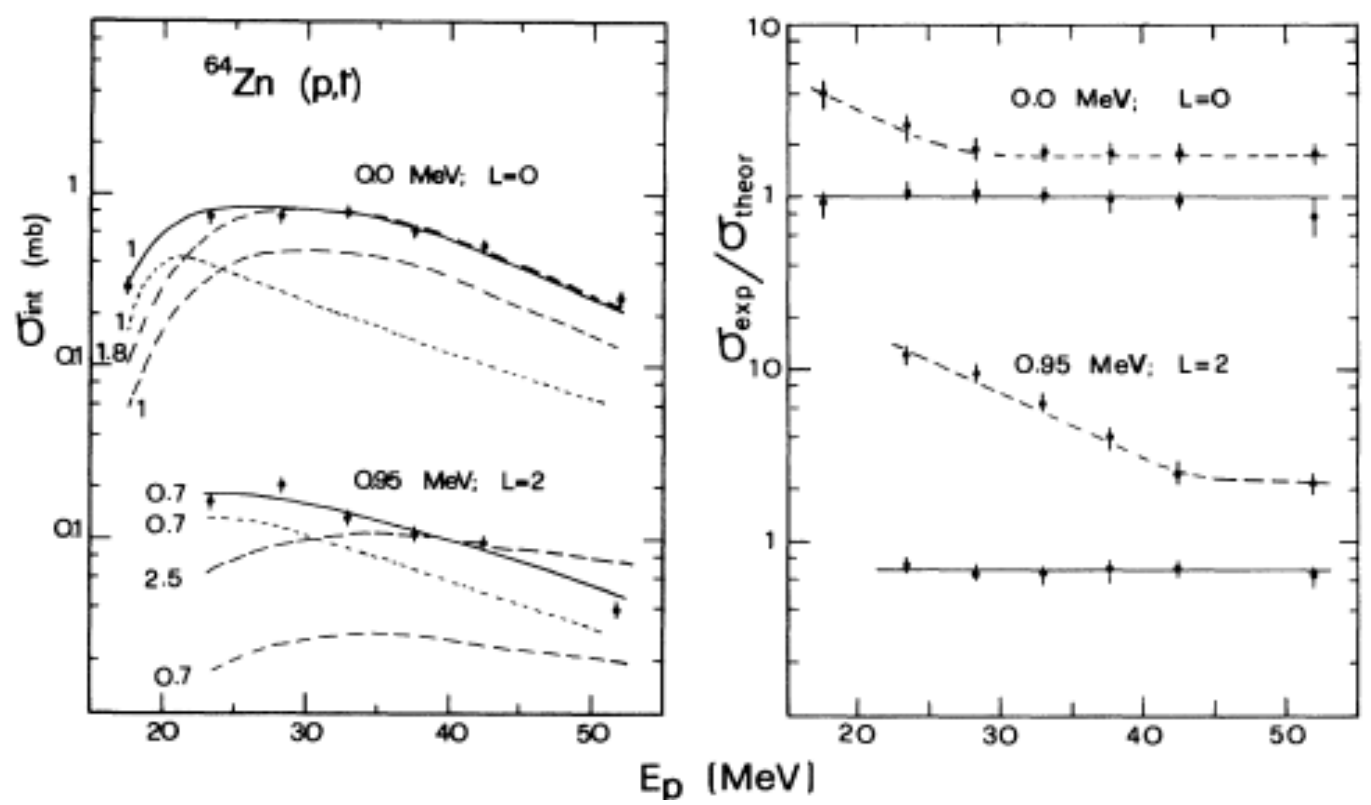

Figure 6 - Data from Ref. [22]. In the left part the integrated cross sections are compared with different theoretical predictions: one-step (long dashed), two-steps (short dashed) and one- plus two-steps (solid lines). The numbers given on the left side of the calculated curves are normalization factors. To evidence better the comparison the one-step curve is reported twice: with the same normalization used in coupled reaction channel calculations and normalized to the experimental data at $42.4 \mathrm{MeV}$. On the right the ratios $\sigma_{\text {exp }} / \sigma t_{\text {heor }}$ are plotted against the incident energy. These ratios were evaluated by fitting the angular distributions with one-step (dashed lines) and one- plus two-step calculations (solid lines).

\section{Design and construction of nuclear instruments}

A large part (nearly the 20\%) of Ileana's scientific publications is related to nuclear instruments. Some of these instruments are already cited above. Also in the years 1980-2008, when she performed experiments in LNL (Legnaro), LNS (Catania), GSI (Darmstadt) and in other laboratories, often in collaboration with Arialdo Moroni, Ileana had in Milan an intense activity addressing the development and to the production of new detection systems. The most recent publications, reporting innovative instruments, are quoted in the contribution of Bassini to this conference. Ileana had always the necessary sensitivity and intuition to involve colleagues and technical personnel of first level, like T. Rossini, during the CISE period, and R. Bassini, A. Bassi, C. Boiano, S. Brambilla, A. Cortesi, M. Malatesta and R. Scardaoni in the next periods.

\section{Conclusions}

The first 25 years of the research activity of Ileana here recalled, have strongly contributed to a progressive understanding of the main features of nuclear reaction processes induced by nucleons up to $45 \mathrm{MeV}$. This research performed in Milano has provided the first systematic and comprehensive experimental work allowing stringent tests to nuclear models. 
The density of the nuclear levels was derived from the evaporative spectra. The widths of the levels in the continuum was deduced observing and understandings the nature of the cross section fluctuations (statistical Ericson fluctuations). Systematic studies on direct processes gave information on two- and three-nucleon transfer reactions and to the multi-steps processes. All these results are still of valuable interest in particular because some of the used approaches will partly inspire the new experimental developments underway for the physics with radioactive beams. Ileana brought, in the collaboration with the Milan group, her scientific culture and her specific and deep knowledge both of the instrumentation and of the theoretical models. She contributed to make the laboratories in Milano and the INFN Milano section well recognized internationally in the field of nuclear physics.

\section{References}

[1] L. Barbanti Silva, C. Bonacini, C. Depietri, I. Iori, G. Lovera, R. Perilli and A. Roveri, On a High Energy Electronic Shower, Il Nuovo Cimento 3 (1956) 1465

[2] N. Dugnani Lonati, U. Facchini, I. Iori, F.G. Houtermann and E. Togiorgi, Study on $\alpha$ Radioactivity in Low Concentrations, Il Nuovo Cimento 7 (1958) 133.

[3] L. Colli, U. Facchini, I Iori, G. Marcazzan, A. Sona and M. Pignanelli, Further Measurements on n,p Reactions at $14 \mathrm{MeV}$, Il Nuovo Cimento 7 (1958) 400.

[4] G. Marcazzan, M. Sona and M. Pignanelli, A Spectrometer for Protons, Il Nuovo Cimento 10 (1958) 155.

[5] K.J. Le Couteaur and D.W. Lang, Neutron evaporation and the level densities in excited nuclei, Nucl. Phys. 18 (1959) 32.

[6] U. Facchini, I. Iori and E. Menichella, Statistical Emission of Neutrons in Reactions Produced by 14 MeV Neutrons, Il Nuovo Cimento 16 (1960) 1109.

[7] E. Erba, U. Facchini and E. Menichella, Statistical Emission in Nuclear Reactions and Nuclear Level Densit, Il Nuovo Cimento 22 (1961) 1237.

[8] T. Ericson, Fluctuations of nuclear cross sections in the "continuum" region. Phys. Rev. Letters 5 (1960) 430 and Ann. of Phys. 23 (1963) 390.

[9] L. Colli, U. Facchini, I. Iori, G.M. Marcazzan, M. Milazzo and F. Tonolini, Fluctuations in the cross section of ${ }^{28} \mathrm{Si}(n, \alpha)^{25} \mathrm{Mg}$ reaction with fast neutrons, Phys. Lett. 9 (1962) 120.

[10] I. Iori, P. Principi and T. Rossini, L'acceleratore elettrostatico da 3.5 MeV del CISE, Energia Nucleare 8 (1961) 770.

[11] Y. Cassagnou, I.Iori, C. Levi, M. Mermaz and L. Papineau, Analysis of fluctuations of integrated cross-sections from ${ }^{27} \mathrm{Al}(d, \alpha)^{25} \mathrm{Mg}$ reactions, Phys. Lett. 8 (1964) 276.

[12] E. Gadioli, I. Iori, M. Mangialaio and G. Pappalardo, Study of the ${ }^{27} \mathrm{Al}(\mathrm{d}, \alpha)^{25} \mathrm{Mg}$ and ${ }^{27} \mathrm{Al}(d, p){ }^{28} \mathrm{Mg}$ reactions at $2 \mathrm{MeV}$ Deuteron Energy, Il Nuovo Cimento 38 (1965) 1105. 
[13] E. Gadioli, I. Iori, A. Marini and M. Sansoni, The level width of atomic nuclei in the continuum energy region: comparison with the statistical-model prevision, Il Nuovo Cimento 44 (1966) 338.

[14] E. Acerbi, M. Castiglioni, G. Dutto, M. Fois, A. Luccio, F. Resmimi, G. Strini, C. Succi and G. Tagliaferri, Initial operation of Milan AVF Cyclotron, Il Nuovo Cimento 35 (1065)1254.

[15] E. Gadioli, I. Iori, N. Molho and L. Zetta, Statistical $\alpha$-Particle Emission from Proton Bombardment of ${ }^{197} A u$, Phys. Rev. C 4 (1971) 1412.

[16] E. Gadioli, I. Iori, N. Molho and L. Zetta, Level Densities of Near Doubly Magic Nuclei from $(p, \alpha)$ Reactions on Bismuth and Lead Isotopes, Il Nuovo Cimento 22 A (1974) 398.

[17] A. Ferrero, E. Gadioli, E. Erba, I. Iori, N. Molho and L. Zetta, $\alpha$ Emission in Proton Induced Reactios, Z. Physik A 293 (1979) 123.

[18] G. Gambarini, I. Iori, S. Micheletti, N. Molho, M. Pignanelli and G. Tagliaferri, Study of (p, $\alpha)$ reactions in light nuclei at $38 \mathrm{MeV}$, Nucl. Phys. A 126 (1969) 562.

[19] P. Guazzoni, I. Iori, S. Micheletti, N. Molho, M. Pignanelli and G. Tagliaferri, On the ( $p, \alpha)$ Direct-Reaction Mechanism in light Nuclei, Il Nuovo Cimento 67 A (1970) 407.

[20] P. Guazzoni, I. Iori, S. Micheletti, N. Molho, M. Pignanelli and G. Semenescu, Energy Dependence of Three-Nucleon Transfer Reactions on Llight Nuclei, Phys. Rev. C 4 (1971) 1092.

[21] M. Pignanelli, S. Micheletti, I. Iori, P. Guazzoni, F.G. Resmini and J.L. Escudié, Energy dependence of two-nucleon transfer reactions on light nuclei, Phys. Rev. C 10 (1974) 445

[22] P. Guazzoni, M. Pignanelli, E. Colombo and F. Crescentini., Energy dependence of transfer reactions on ${ }^{64} \mathrm{Zn}$ and two-step processes, Phys. Rev. C 13 (1976) 1424. 